\title{
Simultaneous Graph Embedding with Bends and Circular Arcs*
}

\author{
Justin Cappos, Alejandro Estrella-Balderrama ${ }^{\star \star}$, \\ J. Joseph Fowler, and Stephen G. Kobourov \\ Department of Computer Science, University of Arizona \\ \{justin, aestrell, jfowler, kobourov\}@cs.arizona.edu
}

\begin{abstract}
We consider the problem of simultaneous embedding of planar graphs. We demonstrate how to simultaneously embed a path and an $n$-level planar graph and how to use radial embeddings for curvilinear simultaneous embeddings of a path and an outerplanar graph. We also show how to use star-shaped levels to find 2-bends per path edge simultaneous embeddings of a path and an outerplanar graph. All embedding algorithms run in $O(n)$ time.
\end{abstract}

\section{Introduction}

Embedding trees and other classes of planar graphs on predetermined pointsets, small integer grids, and levels is motivated by graph layout algorithms and applications in visualizing hierarchical information. Level and radial embeddings can also be used for simultaneous embedding of graphs which are in turn useful in dynamic graph visualization. Simultaneous embedding of planar graphs is also motivated by its relationship with problems of graph and geometric thickness.

A geometric simultaneous embedding of two vertex-labeled planar graphs on $n$ vertices in the $x y$-plane is possible if there exists a labeled point set of size $n$ such that each of the graphs can be realized on that point set (using the vertex-point mapping defined by the labels) with straight-line edges without crossings. For example, any two paths can be simultaneously embedded, while there exist pairs of outerplanar graphs that do not have a simultaneous embedding [3]. Geometric simultaneous embeddings are quite restrictive: pairs of trees and triples of paths may not have such embeddings. Less restrictive versions allow for larger classes of graphs to be embedded without crossings, using few bends per edge [7].

Suppose an $n$-vertex path $P$ is labeled 1 to $n$ from one endpoint to the other. In this paper, we show how to simultaneously embed $P$ with an $n$-vertex planar graph $G$ (also labeled from 1 to $n$ ) that remains planar when the $y$-coordinate of each vertex of $G$ equals its label. We can restrict each vertex of $G$ to lie on the distinct horizontal line, or level, $\ell_{j}=\{(x, j) \mid x \in \mathbb{R}\}$ given by its label $j \in\{1,2, \ldots, n\}$. Such graphs are called level planar graphs with respect to the labeling of $G$. The ability to simultaneously embed $P$ and $G$ in this way depends

\footnotetext{
* This work is supported in part by NSF grants CCF-0545743 and ACR-0222920.

** Partially supported by CONACYT-México.
} 
on the particular labeling of $G$. If $G$ is not level planar for the given labeling, then we give alternative simultaneous embedding techniques provided that $G$ is outerplanar: we retain the straight-line edges for $G$, but relax the edges of $P$ to be either composed of one circular arc each or to have 2 bends per path edge.

\section{$1.1 \quad$ Related Work}

Brass et al. 3] describe linear time algorithms for geometric simultaneous embeddings of pairs of paths, cycles, and caterpillars on an $O(n) \times O(n)$ grid. Geyer et al. [10] show that tree-tree pairs do not always have a geometric simultaneous embedding, and the status of tree-path pairs is open. If bends on the edges are allowed, Erten and Kobourov [7] show that tree-path pairs can be embedded simultaneously using at most one bend per tree edge and no bends of path edges on an $O(n) \times O\left(n^{2}\right)$ grid. Moreover, pairs of planar graphs can be embedded simultaneously using at most 3 bends per edge using an $O\left(n^{2}\right) \times O\left(n^{2}\right)$ grid.

A related problem is that of level planarity, where the goal is to display graphs according to a given hierarchical ordering of the vertices. Such graphs are called level planar graphs [5]. In particular, Jünger and Leipert [11 present a linear time planarity embedding algorithm for level planar graphs using PQ-trees, where the resulting embedding is a set of linear orderings of vertices on each level. Once a graph is determined level planar, Eades et al. 6] can produce a straightline drawing in $O(|V|)$ time, though it may require exponential area. In [8, a characterization of trees that are level planar for any possible labeling of the vertices is given. These trees are called unlabeled level planar (ULP).

\subsection{Our Contributions}

We present results about simultaneous embeddings of pairs of graphs $(P, G)$ on $n$ vertices without crossings, where $P$ is always a path using curvilinear edges or piecewise-linear edges and $G$ is one of several different classes of planar graphs. Our results illustrate the following trade-offs between the class of graphs to which $G$ can belong and the type of edges used for $P$ :

1. If $G$ is level planar, then we show how both $G$ and $P$ can be simultaneously embedded with straight-line edges in $O(n)$ time.

2. If $G$ is outerplanar, we show how to find a plane drawing for $G$ simultaneously with a drawing for $P$ that uses one circular arc per edge in $O(n)$ time.

3. If $G$ is outerplanar and piecewise-linear edges are desirable, we show how to obtain a plane drawing for $G$ simultaneously with a drawing for $P$ that uses two bends per edge in $O(n)$ time.

Table 1 summarizes our current results regarding simultaneous embedding of pairs of planar graphs and relevant previous results. Full details are given in [4]. 
Table 1. Summary of results. ULP here stands for unlabeled level planar, as defined above.

\begin{tabular}{|l|l|l|l|l|}
\hline Pair $(P, G)$ & Edges in $P$ & Edges in $G$ & Condition & Reference \\
\hline \hline (Path, Path) & 0 bends & 0 bends & none & {$[\underline{3}$} \\
\hline (Path, Tree) & 0 bends & 1 bend & none & {[} \\
\hline (Path, Planar) & 0 bends & 0 bends & $G$ is ULP & Theorem [1 \\
\hline (Path, Planar) & 0 bends & 0 bends & $G$ level planar w.r.t. $P$ & Theorem [2] \\
\hline (Path, Outerplanar) & circular arcs & 0 bends & none & Theorem [3] \\
\hline (Path, Outerplanar) & 2 bends & 0 bends & none & Theorem [8] \\
\hline (Path, Tree) & 0 bends & 0 bends & none & Open \\
\hline
\end{tabular}

\section{Geometric Simultaneous Embedding}

We try to use standard notation when discussing level graphs while focusing on the aspects of level graphs that give us simultaneous embeddings using straightline segments. In doing so, we omit from our definitions certain properties of level graphs that are not directly relevant to our problem domain.

Let $G(V, E)$ be an $n$-vertex undirected graph with a labeling $\mathcal{L}: V \stackrel{1: 1}{\underset{\text { onto }}{\longrightarrow}}$ $\{1,2, \ldots, n\}$, which induces a level graph $G(V, E, \phi)$ with a bijective level assignment $\phi=\mathcal{L}$ onto $n$ levels. A vertex $v$ in $V$ is a $j$-level vertex if $\phi(v)=j$. A level drawing is level planar if it admits a plane drawing such that each $j$-level vertex $v$ can be embedded onto the horizontal line $\ell_{j}=\{(x, j) \mid x \in \mathbb{R}\}$. A level graph is level planar if it has a level drawing.

While any planar graph $G$ admits some labeling for which it is level planar, only some planar graphs are level planar regardless of the labeling used. Such graphs are called unlabeled level planar (ULP) [8] and they can be characterized in terms of a pair of forbidden subtrees; see Fig. 1. Moreover, the linear-time recognition and embedding algorithms for ULP trees yield a straightforward way to simultaneously embed an $n$-vertex path and an $n$-vertex ULP tree as illustrated in the following theorem.

Theorem 1. A geometric simultaneous embedding of an n-vertex graph $G$ and an $n$-vertex path $P$ can be computed in $O(n)$ time, provided $G$ is ULP.

Proof. Label the vertices of $P$ sequentially 1 to $n$, and label the vertices of $G$ so that $\mathcal{L}=\phi$ for any $n$-level bijective assignment $\phi$ of the vertices in $G$ and $P$. If $G$ happens to be a ULP tree, it is either a caterpillar, radius- 2 star, or degree-3 spider, each of which has an $O(n)$ time algorithm 8 to produce a compact straight-line level planar drawing of $G$. If $G$ is not a tree, the $O(n)$ time algorithm of Eades et al. 6 can provide a planar straight-line drawing of $G$ with level assignment $\phi=\mathcal{L}$. Regardless, the $y$-coordinate of each $j$-vertex of $G$ matches its label $j$ in a level drawing of $G$. Then we draw the path $P$ in a $y$-monotone fashion zig-zagging upward from one level to the next in $O(n)$ time. This completes our geometric $O(n)$ time simultaneous embedding of $P$ and $G$ since no path edges of $P$ can cross given its $y$-monotone nature. 


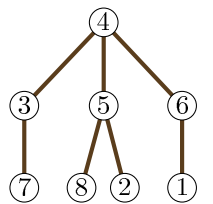

(a)

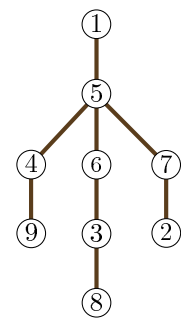

(b)

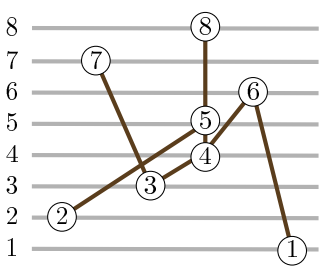

(c)

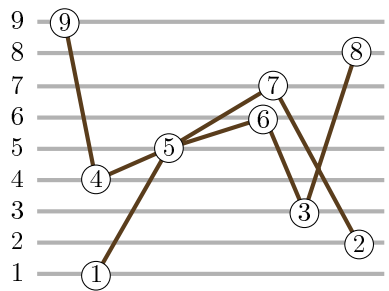

(d)

Fig. 1. Two forbidden trees $T_{1}$ in (a) and $T_{2}$ in (b) that fully characterize ULP trees, and their embeddings with crossings in (c) and (d), respectively, for the given labelings.

The requirement for $G$ to be ULP is overly restrictive. One can use the same approach to simultaneously embed a planar graph $G$ and a path $P$, provided that $G$ is level planar with respect to the labeling induced by $P$. This gives the following theorem.

Theorem 2. A geometric simultaneous embedding of an n-vertex graph $G$ and an n-vertex path $P$ can be computed in $O(n)$ time, provided $G$ is level planar with respect to the labeling given by $P$.

The disadvantage of this approach to simultaneously embed a path and a planar graph is that most planar graphs, including most trees, are not level planar for some labeling. This is not surprising since it is a strong restriction to have a predetermined order of the $y$-coordinates of the vertices. What is surprising, however, is that introducing curvature to the levels, by using circles in lieu of horizontal lines, is enough to allow us to embed all trees and outerplanar graphs with circular arcs for path edges. We show this in the next section.

\section{Simultaneous Embedding with Curves}

This section combines straight-line embeddings of outerplanar graphs with paths consisting of circular arcs to produce curvilinear simultaneous embeddings. First, we describe how to obtain a plane drawing of an $n$-vertex outerplanar graph $G$ on a set of concentric circles such that each vertex lies on a distinct circle, determined by the labeling of $G$. We then use this straight-line crossings-free drawing of $G$ to simultaneously embed $G$ with an $n$-vertex path $P$, such that each path edge consists of a circular arc that lies between adjacent concentric circles. This will give the primary theorem of this section.

Theorem 3. An n-vertex outerplanar graph $G$ can be simultaneously embedded with an $n$-vertex path $P$ in $O(n)$ time such that $G$ forms a plane drawing and $P$ is drawn without crossings using one circular arc per edge. 


\subsection{Embedding an Outerplanar Graph on Concentric Circles}

In this section, we describe how to embed a radial plane drawing of an labeled $n$-vertex outerplanar graph $G$ on a set of distinct $n$ concentric circles using the labeling of $G$. This is similar to a radial level planar embedding on $n$ radial levels, i.e., circles, except that we are using straight lines instead of radially monotone polylines for edges. However, as straight-line edges are not necessarily radially monotone, the radial level planarity test and embeddings algorithms of Bachmaier et al. 1] cannot be directly applied here. Rather, we use the following result from Bose [2].

Theorem 4. (Theorem 6.2 of [2]) If the input point set $\mathcal{P}$ is in convex position then $O(n)$ time and space is sufficient to straight-line embed $G$ into $\mathcal{P}$.

Using this theorem we can obtain our linear time radial straight-line embedding of $G$ onto $n$ concentric circles given by the next lemma.

Lemma 5. Given a set $\mathcal{C}$ of $n$ concentric circles $\left\{C_{1}, C_{2}, \ldots, C_{n}\right\}$ centered at the origin o with monotonically increasing radii $r_{1}, r_{2}, \ldots, r_{n}$, it is a sufficient condition that $\frac{r_{1}}{r_{n}}>\cos \frac{2 \pi}{n}$ in order to obtain in $O(n)$ time a radial plane drawing of an $n$-vertex outerplanar graph $G$ with vertices labeled 1 to $n$ such that each vertex with label $j$ is embedded on circle $C_{j}$.

Proof Sketch: Our strategy is to first embed $G$ using straight-line edges onto a circle $C$ with radius $r$ centered at the origin $o$ without crossings in $O(n)$ time via Theorem 4. We want the vertices of $G$ evenly distributed along the circle, i.e., on a point set $\mathcal{P}$ such that there is a point $p_{i}$ in $\mathcal{P}$ at a distance $r$ from the origin $o$, i.e., $\left|\overline{o p_{i}}\right|=r$, at every radian angle $\theta_{k}=(k-1) \frac{2 \pi}{n}$, where $\theta_{k}$ is the angle $\angle p_{1} o p_{k}$ for $k=1,2, \ldots, n$. Clearly, $\mathcal{P}$ forms a convex set; see Fig. 2 (b).

Then we perturb the vertices in $O(n)$ time in a radial direction so that each one lies on its own circle according to the labeling of $G$, i.e., a vertex $v$ labeled $j$ is placed on $C_{j}$ where $C_{n}=C$. Finally, we determine that if our perturbation is sufficiently small, i.e., $\frac{r_{1}}{r_{n}}>\cos \frac{2 \pi}{n}$, then the radial drawing remains free of crossings. We do this by perturbing the point set $\mathcal{P}$ to match the new locations of the vertices of $G$. We call this perturbed point set $\mathcal{P}^{\prime}$; see Fig. 2(c).

We note that while Theorem 4 works for any convex point set $\mathcal{P}$, which vertex is embedded at which point of $\mathcal{P}$ is determined by the algorithm. We can show that when rerunning the algorithm on point set $\mathcal{P}^{\prime}$ instead of $\mathcal{P}$, it makes the same choices. In order to do that it suffices to show that when we perturb the point set $\mathcal{P}$ to $\mathcal{P}^{\prime}$ the following two conditions hold:

- all the points of $\mathcal{P}^{\prime}$ remain in convex position and

- the order in which each point $p^{\prime}$ of $\mathcal{P}^{\prime}$ sees all the other points $\mathcal{P}^{\prime}-p^{\prime}$ using a radial line sweep centered at $p^{\prime}$ remains the same.

Since the points $\mathcal{P}$ are uniformly distributed over all radial angles, retaining the convexity of $\mathcal{P}^{\prime}$ also achieves the condition of retaining relative positioning provided that the points of $\mathcal{P}^{\prime}$ are only perturbed in a radial direction. Next, we 


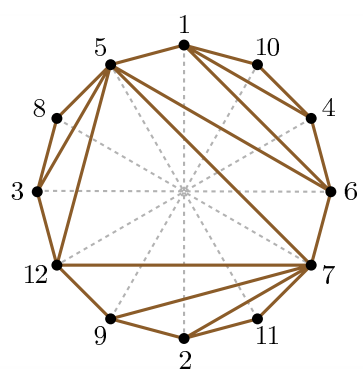

(a)

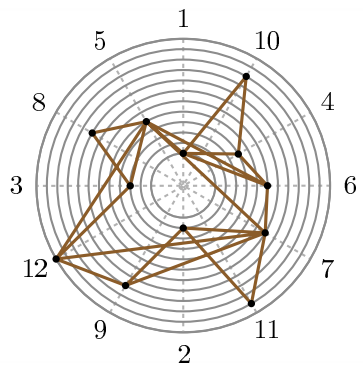

(d)

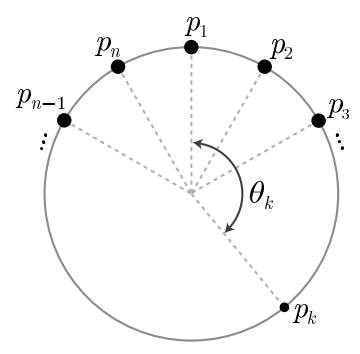

(b)

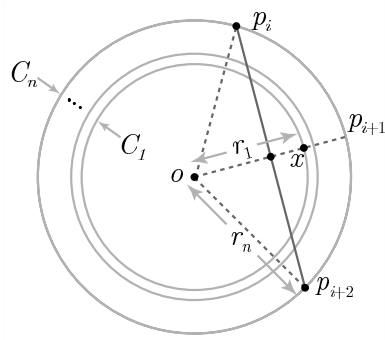

(e)

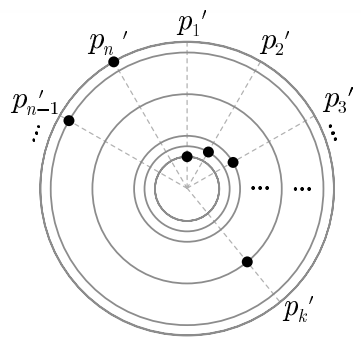

(c)

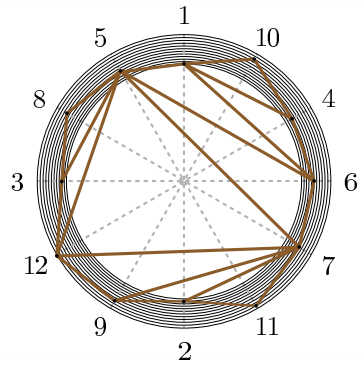

(f)

Fig. 2. The vertices of a 12 -vertex outerplanar graph $G$ are embedded on a circle in (a). This embedding follows the general point set $\mathcal{P}$ given in (b). The points are then perturbed radially inward so that each vertex with label $j$ lies on $C_{j}$ for $j=1,2, \ldots, 12$, where $C_{12}$ is the outermost circle, yielding point set $\mathcal{P}^{\prime}$ in (c). Drawing $G$ on $\mathcal{P}^{\prime}$ gives (d). Crossings are avoided by restricting the ratio between the radii of $C_{1}$ to $C_{n}$ in (e) giving (f).

show that $\frac{r_{1}}{r_{n}}>\cos \frac{2 \pi}{n}$ is a sufficient condition in maintaining the convexity and same convex hull of $\mathcal{P}$ when perturbing the points to $\mathcal{P}^{\prime}$.

Perturbing the point $p_{i+1}$ of $\mathcal{P}$ to lie on the innermost circle $C_{1}$, while letting its neighboring points $p_{i}$ and $p_{i+2}$ along the convex hull of $\mathcal{P}$, remain on $C=C_{n}$, gives a worst case in terms of affecting the convexity of $\mathcal{P}$. Fig. 2(e) illustrates this. Let $x$ denote the midpoint of $\overline{p_{i} p_{i+2}}$. In order for $p_{i+1}$ to remain on the convex hull of $\mathcal{P}$, it is sufficient that the distance from $o$ to $x$ is less than $r_{1}$, the radius of the innermost circle $C_{1}$. Since the angle $\angle x o p_{i}$ is $\frac{2 \pi}{n}$, if the ratio of $\frac{r_{1}}{r_{n}}>\frac{|\overline{o x}|}{r_{n}}=\cos \frac{2 \pi}{n}$, then $p_{i+1}$ will lie in the outer half-plane formed by the line passing through $p_{i}$ and $p_{i+2}$, i.e., the half-plane not containing the origin $o$.

\subsection{Embedding a Path of Circular Arcs Between Concentric Circles}

From Lemma 5, we have that given $n$ distinct concentric circles $C_{1}, C_{2}, \ldots, C_{n}$ of monotonically increasing radii $r_{1}, r_{2}, \ldots, r_{n}$, we can create a plane drawing of any $n$-vertex outerplanar graph $G(V, E)$ with labeling $\mathcal{L}: V \underset{\text { onto }}{\stackrel{1: 1}{\longrightarrow}}\{1,2, \ldots, n\}$ 


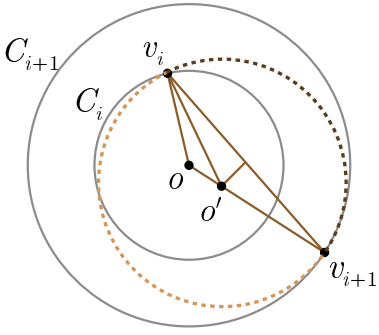

(a)

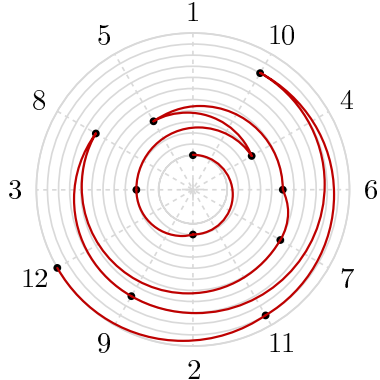

(b)

Fig. 3. Routing one circular arc per edge so that fits inside two consecutive concentric circles is in (a). The concentric circles are centered at $o$ where $o^{\prime}$ is the center of a circle that gives a curve connecting $v_{i}$ and $v_{i+1}$ that stays within the annulus defined by $C_{i}$ and $C_{i+1}$. An example of this is given in (b) for the outerplanar graph from Fig. 2(a).

such that $v \in C_{\mathcal{L}(v)}$ for all $v \in V$ provided $\frac{r_{1}}{r_{n}}>\cos \frac{2 \pi}{n}$. Here we assume the $n$-vertex path $P$ is labeled sequentially 1 to $n$. We show how to route the edges of the path using exactly one circular arc per path edge so that the arcs of $P$ form a radially monotone polyline, which implies that no two circular arcs intersect.

Lemma 6. A radially monotonically increasing crossings-free drawing of an $n$-vertex path $P(V, E)$ with vertex set $V=\left\{v_{1}, v_{2}, \ldots, v_{n}\right\}$ and edge set $E=$ $\left\{\left(v_{1}, v_{2}\right),\left(v_{2}, v_{3}\right), \ldots,\left(v_{n-1}, v_{n}\right)\right\}$ can be realized on $n$ concentric circles $C_{1}, C_{2}$, $\ldots, C_{n}$, where $v_{i} \in C_{i}$ for $1,2, \ldots, n$ with one circular arc per edge.

Proof. It suffices to show that one circular arc always can be used to connect two consecutive vertices on the path, $v_{i}$ and $v_{i+1}$, such that the arc lies strictly outside circle $C_{i}$ and inside circle $C_{i+1}$ except for the end points of the arc at $v_{i}$ and $v_{i+1}$; see Fig. 3(a). Let $o$ be center of the circles. We compute $o^{\prime}$, the center of the circle that forms the desired circular arc connecting $v_{i}$ and $v_{i+1}$ as follows. The center $o^{\prime}$ is the intersection of the perpendicular bisector of $\overline{v_{i} v_{i+1}}$ and the line segment $\overline{o v_{i+1}}$. The radius of the circle centered at $o^{\prime}$ is given by the distance from $o^{\prime}$ to $v_{i}$. The shorter circular arc between $v_{i}$ and $v_{i+1}$ connects the two vertices and is located in the annulus between circles $C_{i}$ and $C_{i+1}$. Furthermore, the distance from $c$ to any point along the arc from $v_{i}$ to $v_{i+1}$ is monotonically increasing. Therefore, the entire path $P$ can be realized as a radially monotone polyline, implying no edge crossings, using one circular arc per edge; see Fig. 3(b).

Lemma 5 together with Lemma 6 gives us Theorem 3 . However, as noted, this only works when we can restrict the radii of the concentric circles to be in a small range. One might wonder whether it is possible to use radially uniform concentric circles instead. The next subsection shows this is not the case. 


\subsection{Trees on Radially Uniform Concentric Circles}

In this section we give an example of a 406-vertex tree with a labeling from 1 to 406 that cannot be straight-line embedded on a set of 406 radially uniform concentric circles such that each vertex lies on its respective circle.

Lemma 7. There exists an 406-vertex tree $T(V, E)$ with labeling $\mathcal{L}: V \underset{\text { onto }}{\stackrel{1: 1}{\longrightarrow}}$ $\{1,2, \ldots 406\}$ that cannot be straight-line embedded on a set $\mathcal{C}$ of 406 radially uniform concentric circles $\left\{C_{1}, C_{2}, \ldots, C_{n}\right\}$ centered at o with radii $r_{1}, r_{2}, \ldots, r_{406}$ such that $r_{i}=(i-1) \Delta$ for $i=1,2, \ldots, 406$ for any $\Delta>0$, where each vertex with label $j$ is embedded on circle $C_{j}$.

Proof. Here we use the ULP forbidden tree $T_{1}$ with 8 vertices from Fig. 1(a) to construct a 406 vertex tree $T$ with root $x$ which has 45 subtrees of 9 vertices each; see Fig. 过(a). Each of the 45 neighbors of $x$ is a degree- 2 vertex connected to a copy of $T_{1}$. We start by labeling $x$ with 1 placing it on $C_{1}$, which has radius 0 , so $x$ must be embedded at the center $o$.

Then we label each of its 45 neighbors with $362,363, \ldots, 406$ so that at least one subtree, which is a copy of $T_{1}$, call it $T_{1}^{\prime}$, must lie within the radian angle $\frac{2 \pi}{45}$. W.l.o.g we assume that this sector is centered along a vertical line passing through the center $o$ since we can rotate the drawing of $T$ as needed.

Within this narrow sector, we observe that the tangents to the circles do no intersect any other circle; see Fig. 4(b). This is because the radius of $r_{i-1}$ is strictly less than $r_{i} \cos \frac{2 \pi}{2 \cdot 45}=r_{i} \cos \frac{\pi}{45}$ for $i=1,2, \ldots, 405$. In particular, $r_{405}=404<r_{406} \cos \frac{\pi}{45}=405 \cdot 0.997564=404.013$.

Then we label the $k^{\text {th }}$ copy of $T_{1}$ with the labels from Fig. 1(c) adding the value of $(k-1) 8+1$ to the labels for $k=1,2, \ldots, 45$. This preserves the $y$-ordering of the labels such that $T_{1}^{\prime}$ (the copy of $T_{1}$ lying strictly within the radian angle

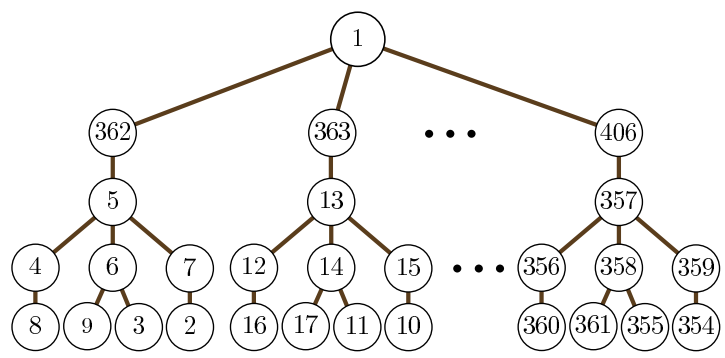

(a)

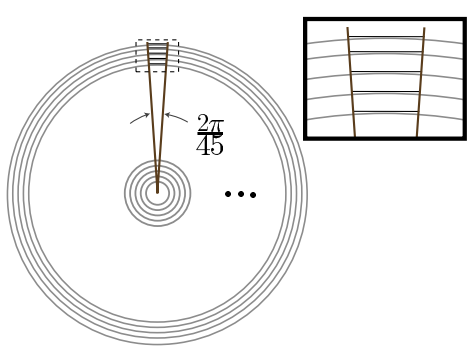

(b)

Fig. 4. The 406-vertex tree in (a) cannot be drawn on radially uniform concentric circles since there must exist one subtree that is a copy of $T_{1}$ from Fig. 1(a) that fully resides in a sector such that tangents of circles do not intersect any other circle. We can rotate this sector so that it lies directly above $o$ so that any vertices placed on the concentric circular arc in this sector must have strictly increasing $y$-coordinates as shown in (b). 
$\frac{2 \pi}{45}$ sector) must have strictly increasing $y$-coordinates. Hence, if $T_{1}^{\prime}$ could be embedded on its circles, then it could be level planarly embedded, which is not the case for the given labeling of $T_{1}^{\prime}$. The inability to level planarly embed $T_{1}^{\prime}$ forbids a straight-line embedding of $T$.

\section{Simultaneous Embedding with Bends}

This section combines straight-line embeddings of outerplanar graphs with paths whose edges are drawn with bends. We introduce the notion of star-shaped levels which allows us to obtain the main result of this section:

Theorem 8. An n-vertex outerplanar graph $G$ can be simultaneously embedded with an $n$-vertex path $P$ in $O(n)$ time such that $G$ forms a plane drawing and $P$ is drawn without crossings with at most 2 bends per path edge.

\subsection{Projecting a 3D Outerplanar Graph onto the 2D $x y$-Plane}

We start by using the following theorem to get a 3-dimensional embedding of an outerplanar graph onto a 3-side prism.

Theorem 9. (Felsner, Liotta and Wismath [9]) Every outerplanar graph $G(V, E)$ with $n$ vertices admits a crossings-free straight-line grid drawing in three dimensions in optimal $O(n)$ volume that can be computed in $O(n)$ time and with the vertices of $G$ drawn on the grid points of a prism.

The ability to do this projection can be used to give our next lemma.

Lemma 10. There exists a projection of a 3-dimensional outerplanar graph $G$ on a 3-sided regular prism onto the xy-plane that preserves the number of straight-line edge crossings of $G$.

Proof Sketch: The embedding of [9] uses the shortest-path distance from some arbitrary root vertex $r$ to every other vertex in the outerplanar graph $G$. Let $d_{\text {mod } 3}(v)$ denote the shortest-path distance from $r$ modulo 3 . Fig. 5 gives an example of an outerplanar graph to be used in illustrating the embedding on star-shaped levels. The 3-dimensional regular prism used for this embedding can be visualized as standing vertically on a triangular base in the $x y$-plane in which the vertical edges are numbered 0,1 , and 2 in clockwise order looking down from the positive $z$-direction. Then $G$ is "wrapped" around the prism such that each vertex $v$ is embedded along the edge $d_{\bmod 3}(v)$ of the prism; see Fig. 6 .

We will use the prism to construct the star-shaped levels. Assume that the base of the prism is an equilateral triangle with side length $\ell$ and that the height of the prism is $3 \ell$. Additionally, let $r=\ell / 2 \sqrt{3}$ be the radius of the circle that is inscribed within the triangle; see Fig. 6(b). We need to shift all the vertices so that they all lie along a fairly narrow band above the distance $2 r$ from the base. This is so that when we "unfold" each vertical side of the prism by laying it flat on the $x y$-plane we do not introduce a crossing; compare Fig. 6(a) to (b). 


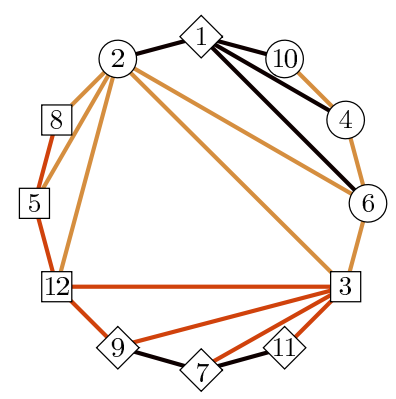

Fig. 5. Outerplanar graph $G$ used as an example for embedding on star-shaped levels. The vertices in this figure and Fig. 6 are in three different shapes (diamonds, circles, and squares) according to whether the vertex is at a shortest-path distance of 0,1 , or 2 modulo 3 from vertex 1 , respectively. The edges are colored one of three colors in a similar fashion.

We pick the side of the rectangular face that is in a $90^{\circ}$ counter-clockwise direction from the prism's base onto which to map the vertices along the prism edge. The positions of the vertices are then mapped directly to their corresponding positions along this projected edge in the $x y$-plane so that the edges on a prism face map directly to straight-line segments in the $x y$-plane.

There is the possibility that extra crossings will be introduced between edges incident to the same pair of prism edges in which one or both of the endpoints

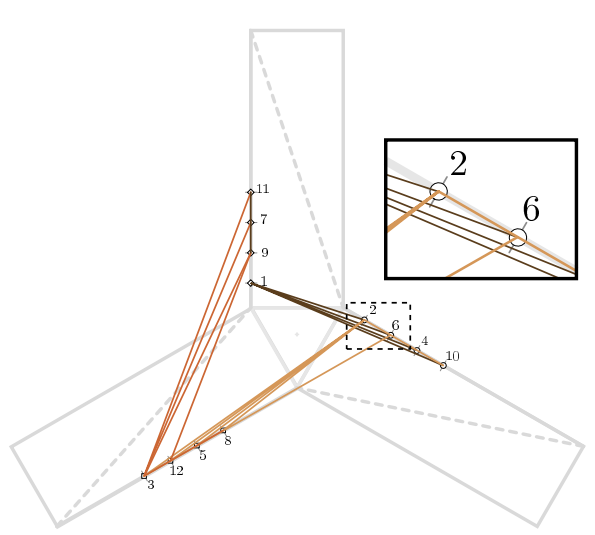

(a)

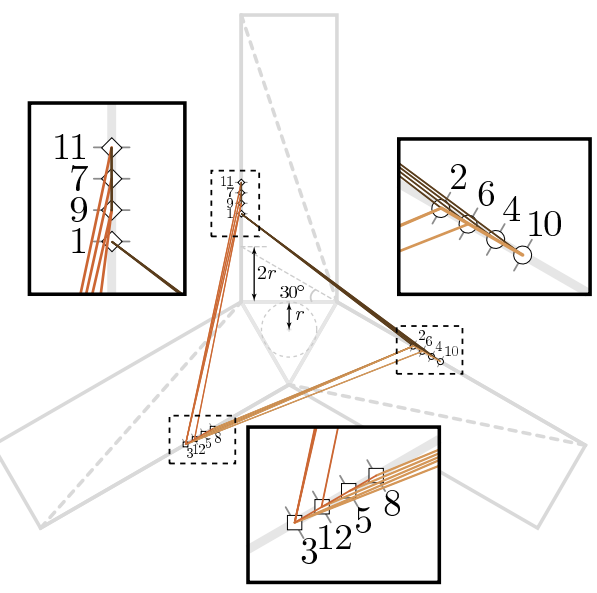

(b)

Fig. 6. Outerplanar graph $G$ from Fig. 5 projected onto the $x y$-plane from its 3dimensional embedding. In (a) there are crossings since the vertices are not above the $2 r$ threshold, where $r$ is radius of the circumscribed circle of the base of the prism. In (b) there are no crossings since all the vertices achieve this threshold. 
lie within a distance of $2 r$ from this base. Once all the endpoints lie above this threshold, which is the point at which an extension of an adjacent prism edge at an angle of $120^{\circ}$ would intersect the edge in the $x y$-plane, then no crossings can occur. This is illustrated by projecting the outerplanar graph $G$ from Fig. 5 onto the $x y$-plane in which all the vertices are not above this threshold in Fig. 6(a), but achieve this $2 r$ threshold in Fig. 6(b) eliminating all crossings.

\subsection{Simultaneous Embedding Using Star-Shaped Levels}

In this section we show how to use the star-shaped levels generated by the outerplanar graph $G$ from the previous section to simultaneously embed a path $P$ with exactly 2 bends per edge giving our next lemma.

Lemma 11. There exists a 2-bend per path edge crossings-free drawing of a path $P$ using star-shaped levels for any vertex labeling.

Proof Sketch: Let $n_{i}$ be the number of vertices along the $i^{\text {th }}$ prism edge (for $i \in\{0,1,2\}$, the shortest-path distance of the vertices from $r$ modulo 3 ) where $n_{\text {max }}=\max \left\{n_{0}, n_{1}, n_{2}\right\}$ is then the total number of star-shaped levels required for this simultaneous embedding. Next we need to perturb the $n_{i}$ vertices that lie along edge $i$ (after performing the above projection to the $x y$-plane) to lie along one of $n_{\max }$ closely adjacent nested star-shaped levels. How close these levels need to be is given by the subsequent lemma. Each star-shaped level has 6 sides and is a scaled-down version of the outermost level (w.r.t. the center of the circle circumscribed within the triangular prism base). This includes the 3 prism edges projected onto the $x y$-plane, and the 3 edges that each connect the top of one projected prism edge to the bottom of the next in a clockwise direction, termed connecting edges; see the dashed 3-pointed star in Fig. 7

When perturbing the vertices, we are careful to move a vertex in a direction perpendicular to the prism edge (as well as all of the adjacent edges of the nested levels) on which it resided. The problem with perturbing the vertices too much is the introduction of crossings in $G$. For example, the outerplanar graph $G$ in Fig. 5 is shown in Fig. 7 on a set of star-shaped levels in which the levels are not spaced sufficiently close enough, resulting in several crossings.

The vertices are placed clockwise from outermost to innermost star-shaped level by the order given by the path labeling. However, in order to be able to route the path back and forth between vertices that alternate back and forth between adjacent sides of the prism, an extra connecting edge needs to be inserted to lie half-way between every pair of adjacent connecting edges. Since we need one for each level, an extra connecting edge also needs to be added to lie just interior to the $n_{\max }{ }^{\text {th }}$ innermost connecting edge. In Fig. 7 these are shown as dashed line segments. We denote the levels along which the vertices lie as regular levels, which are depicted in Fig. 7 with solid gray edges, and the in-between levels as half-levels consisting of dashed edges.

The rule then for going from one vertex $u$ to the next vertex $v$ is that if going clockwise, then $v$ 's connecting regular level's edge is used, otherwise, its 


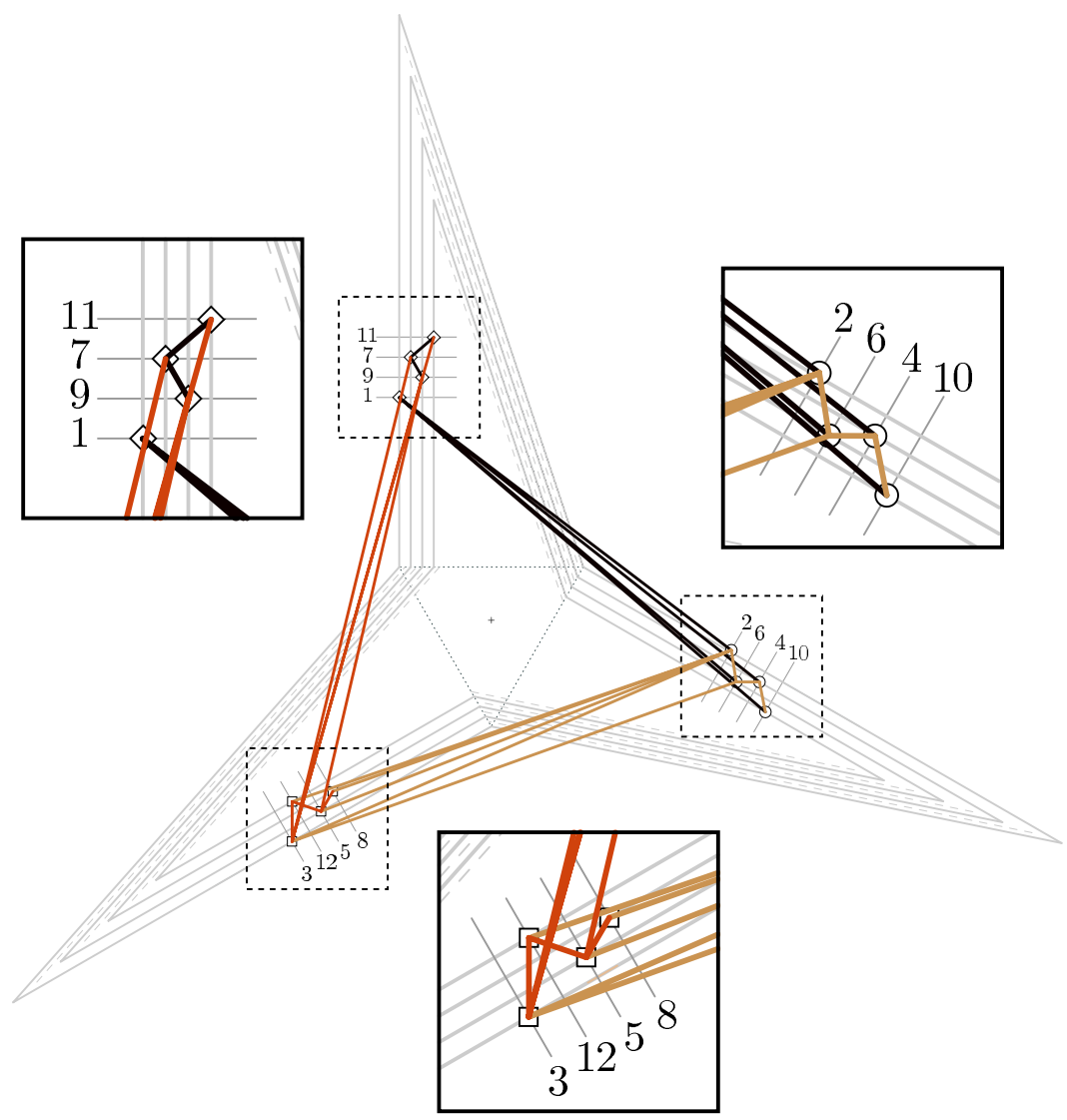

Fig. 7. Outerplanar graph $G$ from Fig. 5 embedded onto the star-shaped levels

connecting half-level's edge is used. Hence, if going clockwise, 2 bends are introduced at each endpoint of the connecting regular level's edge corresponding to the destination vertex. Otherwise, 2 bends are introduced at each endpoint of the connecting half-level's edge of the destination vertex.

As was the case with circular arcs, making the star-shaped levels sufficiently close for a given outerplanar graph $G$, as well as an additional technical condition of placing the vertices of $G$ in close proximity along the middle portion of the prism edge, avoids crossings as given by our final lemma. The proof of this lemma (along with a detailed description of the geometry of the star-shaped levels) is included in the technical report [4].

Lemma 12. Let $G$ be an $n$-vertex outerplanar graph. Let $\delta$ be the maximum separation between two vertices of $G$ along the same prism edge before perturbing vertices onto the other star-shaped levels, and let $\Delta$ be the maximum separation between the nested star-shaped levels. Then $G$ will be crossings-free when embedded onto the star-shaped levels provided $\Delta<\frac{\ell}{10 n}$ and $\delta<\frac{\ell}{(n-1)^{2}}$. 


\section{Conclusions and Open Problems}

We presented results in simultaneous embeddings of path and outerplanar graphs with circular arc edges or a small number of bends. Other open problems include:

1. Do all tree-path pairs have geometric simultaneous embedding?

2. What is the complexity of determining whether two planar graphs admit a geometric simultaneous embedding?

3. What is the complexity of determining whether a pair of graphs can be simultaneously embedded?

\section{Acknowledgments}

We would like to thank Jiří Fiala, Ferran Hurtado, and David Wood for the stimulating discussions about this problem.

\section{References}

1. C. Bachmaier, F. J. Brandenburg, and M. Forster. Radial level planarity testing and embedding in linear time. J. Graph Algorithms Appl., 9(1):53-97, 2005.

2. P. Bose. On embedding an outer-planar graph in a point set. CGTA: Computational Geometry: Theory and Applications, 23(3):303-312, 2002.

3. P. Brass, E. Cenek, C. A. Duncan, A. Efrat, C. Erten, D. Ismailescu, S. G. Kobourov, A. Lubiw, and J. S. B. Mitchell. On simultaneous graph embedding. In 8th Workshop on Algorithms and Data Structures, pages 243-255, 2003.

4. J. Cappos, A. Estrella-Balderrama, J. J. Fowler, and S. G. Kobourov. Simultaneous graph embedding with bends and circular arcs. Technical Report TR06-02, University of Arizona, 2006.

5. G. Di Battista and E. Nardelli. Hierarchies and planarity theory. IEEE Trans. Systems Man Cybernet., 18(6):1035-1046 (1989), 1988.

6. P. Eades, Q. Feng, X. Lin, and H. Nagamochi. Straight-line drawing algorithms for hierarchical graphs and clustered graphs. Algorithmica, 44(1):1-32, 2006.

7. C. Erten and S. G. Kobourov. Simultaneous embedding of planar graphs with few bends. In 12th Symposium on Graph Drawing (GD), pages 195-205, 2004.

8. A. Estrella-Balderrama, J. J. Fowler, and S. G. Kobourov. Characterization of unlabeled level planar trees. Manuscript accepted by 14th Symposium on Graph Drawing, 2006.

9. S. Felsner, G. Liotta, and S. Wismath. Straight-line drawings on restricted integer grids in two and three dimensions. Journal of Graph Algorithms and Applications, 7(4):363-398, 2003.

10. M. Geyer, M. Kaufmann, and I. Vrto. Two trees which are self-intersecting when drawn simultaneously. In 13th Symposium on Graph Drawing, pages 201-210, 2005.

11. M. Jünger and S. Leipert. Level planar embedding in linear time. J. Graph Algorithms Appl., 6(1):67-113, 2002. 